\title{
68. Studies on Discogenic Pain
}

\author{
Jiro Suzuki, Shun-ichi Inoue, Masataka Tateiwa, \\ Haruo Tsuji, Atsuchi Saito, Minoru Mituhashi \\ Shigeru Taniguchi, Mutsumi Komatsuzaki, \\ Keiichi Tsuchiya, Kenta Murayama, \\ and Hirosuke SuzUKI \\ Department of Orthopaedic Surgery, School of Medicine, Chiba University
}

Since 1956, discography was achieved on over 700 intervertebral discs. Deep low back pain and sciatica appeared by the injection of Plraceton (contrast medium) into the intervertebral discs. On the analysis of those discogram, complaints at discography were noticed not only in the cases of posteriorly protruded discs but also in the cases of anteriorly protruded discs and non-protruded degenerative discs. Discogram on the cases of persistnt apophysis, Schmorl's node, and spondylosis deformans showed that the pathogenesis of those diseases was also "discopathy". These facts seemed to imply the existence of discogenic pain.

Electromyographical investigations at the back muscle and the muscles of the lower extremity were achieved with the injection of Pyraceton into the intervertebral discs. Spontaneous discharge at discography was noticed on the sacrospinal, anterior tibial, and calf muscles even in the cases of anteriorly protruded disc. The apparance of spontaneous discharge at discography was proportionate to the degenerative changes of the discs.

The measurement of intra-discal pressure (discometry) was done. Intradiscal pressure without loading was lower at the degenerative discs. The increasing rate of intradiscal pressure with the injection of pyreaceton was also lower at the degenerative discs. It was thought on the comparison with the pathological investigation of the excised discs and cartilaginous plate by operation, that the leakage of intra-discal pressure and injected materials was involved in the ocurrence of discogenic pain.

Silver-stain and vital-stain by Methylen-blue were achieved on the discs. Free nerve ending and non-encapsulated ending were found out in the outer layer of this annulus fibrosus and in the inner side of the ligaments. Those nerve endings were confirmed electro-physiologically on the decerebrated cats. That is, afferent impulse was brought out at the single fiber of the posterior never root by the electric stimulation in the outer layer of the annulus fibrous of the decerebrated cat.

Net problem is: What kind of stimulus within the disc makes pain on those nerve endings pathological degeneration in the annulus and cartilaginous plate seems to be a kind of stimuli to make pain on the nerve endings. 
Other important stimuli are bio-chemical changes within the disc. The decrease of Hexosamine-collagen ratio and the reversal and the decrease of Glucosamine-Galactosamine ratio on discopathy showed metabolic disturbance in the ground substance within the disc. Out of amino-acids within the disc, Tyrosine, Phenylalanie, and Tryptophane increased on discopathy. It may be that those three amino-acids are concerned in blackish pigmentation which was often noticed within the degnerative disc. Tryptophane is created by way of Serotonin which is the intermediate metabolic product, rolls as chemical transmitter substance within the nervous system, and is suggested as a pain-making substance.

It is of interest to speculate that some substance which is produced in the metabolic pathways within the discs stimulates nerve endings and, then, discogenic pain comes.

\title{
69. Long-term Results of Surgical Treatment of Cervical Spondylosis
}

\author{
Haruo Takamura, Hiroshi Are, Masaharu Sato, Kunio Tashiro, Takashi Owada \\ and Mitsuyuki KorwA \\ Department of Neurosurgery, Hokkaido University School of Medicine
}

In the past 8 years we experienced 232 cases of cervical disc lesions. Although most of these cases were treated conservatively, using cervical collars or medications operative procedures were carried out in 58 cases that were affected severely. These surgical cases consisted of 32 cases with spondylosis, 5 with hard disc, 14 with soft disc and 9 with soft disc accompanied by spondylosis. The operative methods that we used in 32 cases with spondylosis were as follows:

Table 1.

\begin{tabular}{l|c|c}
\hline & total cases & $\begin{array}{c}\text { follow up } \\
\text { cases } \\
\text { (over 1 year) }\end{array}$ \\
\hline 1) laminectomy + cutting of dentate ligamehts & 22 & 13 \\
2) laminectomy + foraminectomy & 7 & 5 \\
3) anterior approach with or without laminectomy & 3 & 22 \\
\hline
\end{tabular}

Long-term results were compared among these operative procedures, concerning cord and root signs. 22 cases underwent the first method (laminectomyt 